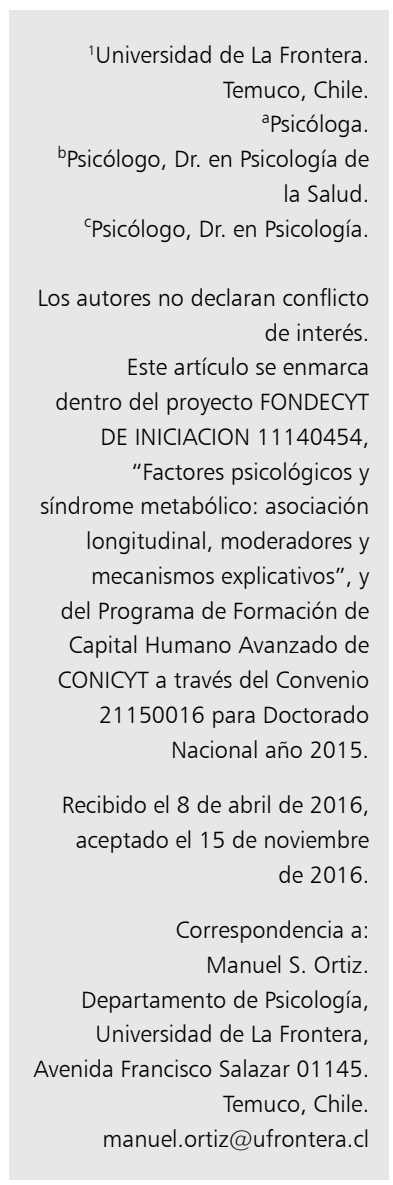

\section{Estigma de obesidad, su impacto en las víctimas y en los Equipos de Salud: una revisión de la literatura}

\author{
DANIELA GÓMEZ-PÉREZ ${ }^{1, a}$, MANUEL S. ORTIZ ${ }^{1, \mathrm{~b}}$, \\ JOSÉ L. SAIZ ${ }^{1, \mathrm{c}}$
}

\section{The effects of weight stigmatization on obese people and health care professionals}

Weight stigmatization is defined as the devaluation of others based on weight, a visible and specific characteristic that cannot be hidden. The consequences of weight stigmatization have been studied in victims and healthcare providers. This narrative literature review describes the consequences of weight stigmatization in victims and healthcare practitioners. According to several studies, high levels of psychological dysfunction in victims coexist with negative stereotypes in healthcare professionals, since the latter are exposed to the same negative media messages about obesity. Thus, weight stigmatization contributes to increase the burden of obesity, since when obese patients perceive that they are discriminated by healthcare professionals, their unhealthy behaviors persist and they have a low adherence to obesity treatment and medical recommendations.

(Rev Med Chile 2017; 145: 1160-1164)

Key words: Discrimination (Psychology); Health Personnel; Obesity; Social Stigma.
E l estigma de obesidad consiste en la devaluación que se hace de un individuo por ser obeso, característica que es visible, particular y que no es posible de ocultar, siendo además, uno de los primeros aspectos que se observa en una persona ${ }^{1}$. Los estudios señalan que quienes son blanco de tal estigmatización tienen pobres resultados en salud mental, mayor enfermedad física, peor bienestar individual, bajo rendimiento académico y dificultades en el acceso a bienes de capital, educación y oportunidades laborales ${ }^{1-3}$, están expuestos a discriminación en diferentes ámbitos los cuales incluyen entornos de salud, trabajo y relaciones interpersonales ${ }^{4}$, constituyéndose por tanto, en un problema relevante con consecuencias psicosociales profundas. Dadas las altas cifras de sobrepeso $(39,3 \%)$ y obesidad $(27,4 \%)$ en Chile $^{5}$, es posible hipotetizar que un porcentaje considerable de la población puede llegar a ser objeto de tal estigmatización.

Las consecuencias psicosociales del estigma por obesidad han sido estudiadas mayoritariamente en quienes son víctimas de tal estigmatización, sin embargo, menor atención se ha puesto en quienes estigmatizan, voluntaria o involuntariamente, por ejemplo, familia, amigos, conocidos, prestadores de servicios, entre otros. Por tal motivo, esta revisión narrativa de la literatura se centra en las consecuencias que el estigma de obesidad tiene tanto en quienes son blanco del estigma, como en el ejercicio profesional de los equipos de salud que interactúan con personas o pacientes obesos.

Este manuscrito podría favorecer la comprensión de este fenómeno e identificación de las consecuencias que tiene en la salud de los individuos obesos. Además, podría contribuir a mejorar las intervenciones que brindan profesionales de la salud a personas obesas, haciéndolos conscientes 
de sus propios estereotipos de obesidad. Para tal efecto, se ha realizado una revisión narrativa de la literatura, analizando e integrando los resultados de estudios pertinentes, de reciente publicación, que incluyen la problemática de estigma de obesidad y que se encuentran disponibles en bases de datos PubMed y PsychINFO. Los criterios de búsqueda fueron obesity stigma, antifat attitude, discrimination, healthcare, overweight and obesity. Se incluyeron artículos cuya fecha de publicación estuviese contenida entre los años 2000 y 2016.

\section{Contextualización general del estigma de obesidad}

El estigma de obesidad se ha investigado tanto en sujetos con obesidad como en sujetos sanos, pretendiendo identificar la percepción que se tiene sobre individuos con obesidad y las diferencias en cuanto a la consideración de aspectos sociodemográficos como la edad, género e identificación étnica ${ }^{6}$. Los estudios son consistentes en demostrar que la estigmatización ocurre hacia hombres y mujeres por igual, no obstante, cuando las víctimas de esta estigmatización son mujeres, ellas refieren peor calidad de vida y bienestar, además de autoestima reducida en comparación a los hombres, quienes reportan problemas asociados al ámbito laboral ${ }^{6,7}$, es decir, las mujeres podrían verse mayormente afectadas cuando son víctimas de este estigma ${ }^{8}$.

Diversas investigaciones sobre estigma de obesidad concluyen que existen más actitudes estigmatizantes cuando hay una mayor atribución de responsabilidad individual de la condición de obesidad $^{9,10}$, cuando a los obesos se le asocian características de personalidad que les impide cambiar patrones conductuales en relación a la alimentación, o cuando se cree que la obesidad es controlable por el sujeto que la padece ${ }^{11-13}$. A lo anterior se suma que la mayoría de las personas desconoce como causa de la obesidad a los factores genéticos involucrados, lo cual aumenta el estigma hacia los sujetos con esa condición, ya que se concibe la obesidad como un defecto moral o asociado exclusivamente a la conducta del individuo ${ }^{11,14}$.

Otro aspecto relevante mencionado en diversas investigaciones es que, a diferencia de lo que ocurre con otros grupos estigmatizados, las personas obesas se estigmatizan a sí mismas tanto como lo hacen otras personas no obesas ${ }^{6,13,15,16}$, teniendo consecuencias negativas en las víctimas y también en las personas con quienes interactúa. Lo anterior incluye a los equipos de salud que intervienen en esta patología, ya que prácticas discriminatorias derivadas del estigma de obesidad podrían dar lugar a la evitación de los entornos de salud y a la baja adherencia terapéutica de la intervención con especialistas ${ }^{3,17,18}$.

\section{Consecuencias del estigma de obesidad en las víctimas}

Las víctimas del estigma reportan que habitualmente reciben comentarios negativos de terceras personas o el entorno hace supuestos negativos respecto de ellos mismos ${ }^{19}$ además de estar expuestos a barreras físicas y al rechazo social ${ }^{20}$. Las personas que tienen sobrepeso o son obesas, sólo por esa condición se enfrentan a inequidades sociales importantes, por ejemplo en el ámbito económico obtienen salarios inferiores por el mismo trabajo en comparación a sujetos con un peso normal, tienen menos oportunidades de ascenso y suelen ser víctimas de despidos injustificados ${ }^{1}$. El estigma de obesidad también se ha asociado con desajuste psicológico en la víctima, vinculándose principalmente con depresión, ansiedad, insatisfacción corporal y baja autoestima ${ }^{2,19-22}$. Incluso, el impacto que la obesidad tiene en la autoestima de la víctima es observable desde etapas tempranas de la vida. Por ejemplo, en un estudio longitudinal realizado con 1.520 niños de 9 y 10 años de edad, se encontró que los niños obesos disminuyeron su autoestima a los 4 años de seguimiento, en comparación a los niños de peso normal ${ }^{23}$.

El impacto que genera la estigmatización en las víctimas es mayor si se considera que este tiene repercusiones en conductas de salud. Es frecuente que las víctimas reporten una mayor ingesta de alimentos, rechazo a la dieta y menor actividad física debido al estigma ${ }^{24-26}$. En efecto, en un estudio con una muestra de 2.449 obesos, $79 \%$ reportó usar la estrategia de ingerir una mayor cantidad de alimentos y sólo $10 \%$ reportó nunca haber utilizado esa estrategia como respuesta al estigma ${ }^{27}$.

En contextos médicos, las personas obesas refieren ser atendidas por períodos de tiempo más breves y reportan una menor calidad en la atención en comparación con sujetos con un índice de masa 
corporal normal $^{1,28}$. El estigma de obesidad genera comportamientos que no contribuyen al control del peso $^{29}$, tales como el retraso o evitación en la búsqueda de prestaciones médicas ${ }^{30,31}$, menor motivación por realizar actividad física ${ }^{32,33}$ o una ingesta calórica superior a la recomendada por los especialistas ${ }^{19,34,35}$. Además, la discriminación derivada del estigma, se ha asociado a presión arterial elevada, lo que pone en un doble riesgo a los obesos ${ }^{28,36,37}$.

\section{Consecuencias del estigma de obesidad en los equipos de salud}

Los profesionales que conforman los equipos de salud están expuestos a los mismos mensajes sociales sobre las personas obesas que los miembros de la población en general, por lo que podrían, sin quererlo, perpetuar el estigma hacia la obesidad ${ }^{38-41}$.

Existe evidencia científica sobre presencia de actitudes desfavorables automáticas, esto es, fuera de control consciente hacia personas que tienen sobrepeso o son obesas, incluso entre profesionales de la salud ${ }^{29}$. Lo anterior alude a sesgos negativos implícitos hacia los obesos, lo cual no necesariamente implica una conducta discriminatoria manifiesta ${ }^{42}$. Por ejemplo, en un estudio se encontró que los profesionales de la salud asociaron la obesidad con estereotipos de personas perezosas y carentes de valor, asumiendo que los obesos tienen características y conductas que contribuyen a su problema, incluyendo dimensiones cognitivas y de valor personal ${ }^{43}$; de hecho, médicos atribuyen características negativas como voluntad débil a pacientes obesos ${ }^{30} y$ aunque tienen menor sesgo negativo implícito en comparación con la población en general ${ }^{42}$, de igual modo esta situación podría contribuir a que pacientes obesos se perciban discriminados. En efecto, un estudio con pacientes de cirugía bariátrica demostró que ellos se sintieron incomprendidos y maltratados por el personal médico y no médico que participó de la intervención $^{44}$. Similarmente, en estudios realizados con enfermeras, se demostró que estas profesionales reportaron creencias y actitudes negativas hacia personas con sobrepeso y obesidad, percibiéndolas como menos exitosas, más incompetentes y con peor salud que personas no obesas ${ }^{45,46}$.

El estigma de obesidad también puede tener consecuencias sobre las recomendaciones entregadas por especialistas hacia personas con sobrepeso u obesidad. En un estudio realizado con médicos, se encontró que $40 \%$ de ellos tuvieron reacciones negativas hacia pacientes obesos, reportando altos niveles de frustración, con la mayoría de ellos, al momento de tratarlos ${ }^{47}$. En otro estudio, médicos que atendían a un alto porcentaje de pacientes con obesidad mórbida, entregaron menos recomendaciones de tratamiento farmacológico o cirugía bariátrica para el manejo del peso ${ }^{48}$.

Asimismo, el estigma de obesidad que tienen los profesionales de la salud podría estar asociado con el tiempo dedicado a los pacientes, la empatía, la calidad de las interacciones, el optimismo respecto a la mejora del paciente y la voluntad e intención de proporcionar ayuda ${ }^{43}$. En relación a lo anterior, se ha reportado que los profesionales de la salud tienden a ofrecer poca o ninguna orientación para el control del peso a sus pacientes, lo que podría relacionarse a las bajas expectativas que tienen en cuanto a los resultados de la intervención o a la asociación de estereotipos negativos sobre obesos en cuanto a su falta de autocontrol ${ }^{49}$. Similarmente, en un estudio se plantea que los profesionales que atienden obesos no recogen un historial clínico completo, ya que la mayoría de las dificultades reportadas por el paciente son atribuidas a la obesidad, limitando la indicación de otros exámenes para comprobar sus hipótesis diagnósticas o realizando exámenes físicos incompletos $^{30}$.

\section{Conclusión}

En Chile existen altas cifras de sobrepeso y obesidad. Lo anterior impone un desafío en materias de salud pública, pues tal como se ha mencionado en este manuscrito, los efectos que tiene el estigma asociado al peso genera consecuencias tanto en la víctima como en los equipos de salud. En relación a los primeros, se ha encontrado un mayor desajuste psicológico, inequidades sociales y dificultades en las relaciones sociales y laborales. En cuanto a las consecuencias en los equipos de salud, se ha encontrado que estos no están ajenos a la estigmatización por obesidad y por tanto suelen tener conductas discriminatorias con pacientes obesos, lo que podría tener implicancias en la intervención y en los resultados esperados en salud. 
Dado que el estigma de obesidad tiene consecuencias conductuales, tanto en la víctima como en los equipos de salud, no considerar esta variable en las estrategias de intervención, podría volverlas menos efectivas, ya que se genera una dinámica circular en términos que el paciente obeso al estar expuesto al estigma afronta este estresor con conductas que son la antítesis del control de peso, y a su vez el equipo médico al tener un sesgo implícito negativo tiene comportamientos que refuerzan el estigma, lo que en su conjunto contribuye a perpetuar la enfermedad. Es importante considerar que además de los factores genéticos y neuroendocrinos involucrados en la obesidad, los factores psicológicos y sociales tienen un rol relevante en la comprensión de este fenómeno y por tanto las intervenciones deben ser multidisciplinarias.

El rol que juegan los equipos médicos en la intervención de la obesidad es fundamental, ya que además de prescribir un tratamiento y entregar orientaciones específicas, podrían por un lado ayudara disminuir la estigmatización de las personas obesas educando a la población general respecto a las causas de la obesidad y por otra parte, al ser conscientes de sus propios estereotipos, podrían favorecer la intervención generando un vínculo con el paciente que rompa con la circularidad antes mencionada.

\section{Referencias}

1. Puhl R, Heuer C. The stigma of obesity: a review and update. Obesity 2009; 17 (5): 941-64.

2. Himmelstein M, Tomiyama A. J. It's Not You, It's Me: Self-Perceptions, Antifat Attitudes, and Stereotyping of Obese Individuals. Soc Psychol Personal Sci 2015; 6 (7): 749-57.

3. Puhl R, Heuer C. Obesity stigma: Important considerations for public health. Am J Public Health 2010; 100 (6): 1019-28.

4. Jackson SE. Obesity, Weight Stigma and Discrimination. Obes Eat Disord 2016; 2 (1): 1-3.

5. Ministerio de Salud Chile. Principales resultados Encuesta Nacional de Salud 2010.

6. Latner J, Stunkard A, Wilson G. Stigmatized students: age, sex, and ethnicity effects in the stigmatization of obesity. Obes Res 2005; 13 (7): 1226-31.

7. Wee C, Davis R, Chiodi S, Huskey K, Hamel M. Sex, Race, and the Adverse Effects of Social Stigma vs. Other Quality of Life Factors Among Primary Care Patients with Moderate to Severe Obesity. J Gen Intern Med 2014; 30 (2): 229-35.

8. Chen EY, Brown M. Obesity stigma in sexual relationships. Obes Res 2005; 13 (8): 1393-7.

9. Schmalz DL, Colistra CM. Obesity Stigma as a Barrier to Healthy Eating Behavior. Top Clin Nutr 2016; 31 (1): 86-94.

10. Brady C. Decreasing Obesity and Obesity Stigma: Socio-Demographic Differences in Beliefs about Causes of and Responsibility for Obesity. Soc Sci 2016; 5 (1): 1-12.

11. Ebneter DS, Latner JD, O’Brien KS. Just world beliefs, causal beliefs, and acquaintance: Associations with stigma toward eating disorders and obesity. Pers Individ Dif 2011; 51 (5): 618-22.

12. Holub SC, Tan CC, Patel SL. Factors associated with mothers' obesity stigma and young children's weight stereotypes. J Appl Dev Psychol 2011; 32 (3): 118-26.

13. Jáuregui I, López I, Montaña M, Morales M. Percepción de la obesidad en jóvenes universitarios y pacientes con trastornos de la conducta alimentaria. Nutr Hosp 2008; 23 (3): 226-33.

14. Brownell KD, Kersh R, Ludwig DS, Post RC, Puhl RM, Schwartz MB, et al. Personal responsibility and obesity: A constructive approach to a controversial issue. Health Aff 2010; 29 (3): 379-87.

15. Latner J, O’Brien K, Durso L, Brinkman L, Macdonald T. Weighing obesity stigma: The relative strength of different forms of bias. Int J Obes 2008; 32 (7): 1145-52.

16. Wang SS, Brownell KD, Wadden TA. The influence of the stigma of obesity on overweight individuals. Int J Obes Relat Disord 2004; 28 (10): 1333-7.

17. MacLean L, Edwards N, Garrard M, Sims-Jones N, Clinton K, Ashley L. Obesity, stigma and public health planning. Health Promot Int 2009; 24 (1): 88-93.

18. Phelan SM, Burgess DJ, Puhl R, Dyrbye LN, Dovidio JF, Yeazel M, et al. The Adverse Effect of Weight Stigma on the Well-Being of Medical Students with Overweight or Obesity: Findings from a National Survey. J Gen Intern Med 2015; 30 (9): 1251-8.

19. Puhl R, Brownell K. Confronting and coping with weight stigma: an investigation of overweight and obese adults. Obesity (Silver Spring) 2006; 14 (10): 1802-15.

20. Friedman KE, Reichmann SK, Costanzo PR, Zelli A, Ashmore JA, Musante GJ, et al. Weight stigmatization and ideological beliefs: Relation to psychological functioning in obese adults. Obes Res 2005; 13 (5): 907-16.

21. Myers A, Rosen JC. Obesity stigmatization and coping: relation to mental health symptoms, body image, and self-esteem. Int J Obes Relat Metab Disord 1999; 23: 221-30. 
22. Murakami JM, Latner JD. Weight acceptance versus body dissatisfaction: Effects on stigma, perceived self-esteem, and perceived psychopathology. Eat Behav 2015; 19: 163-7.

23. Strauss RS. Childhood Obesity and Self-Esteem. Pediatrics 2000; 105 (1): 1-5.

24. Ashmore JA, Friedman KE, Reichmann SK, Musante GJ. Weight-based stigmatization, psychological distress, \& binge eating behavior among obese treatment-seeking adults. Eat Behav 2008; 9 (2): 203-9.

25. Puhl RM, Moss-Racusin CA, Schwartz MB. Internalization of weight bias: Implications for binge eating and emotional well-being. Obesity (Silver Spring) 2007; 15 (1): 19-23.

26. Schvey NA, Puhl RM, Brownell KD. The impact of weight stigma on caloric consumption. Obes (Silver Spring) 2011; 19 (10): 1957-62.

27. Puhl RM, Brownell KD. Confronting and coping with weight stigma: an investigation of overweight and obese adults. Obesity (Silver Spring) 2006; 14 (10): 1802-15.

28. Carr D, Friedman MA. Is obesity stigmatizing? Body weight, perceived discrimination, and psychological well-being in the United States. J Health Soc Behav 2005; 46 (3): 244-59.

29. Fruh SM, Nadglowski J, Hall HR, Davis SL, Crook ED, Zlomke K. Obesity Stigma and Bias. J Nurse Pract 2016; 12 (7): 425-32.

30. Drury CA, Louis M. Exploring the association between body weight, stigma of obesity, and health care avoidance. J Am Acad Nurse Pract 2002; 14 (12): 554-61.

31. Wee C, McCarthy E, Davis R, Phillips R. Screening for cervical and breast cancer: Is obesity an unrecognized barrier to preventive care? Ann Intern Med 2000; 132 (9): 697-704.

32. Vartanian L, Novak S. Internalized societal attitudes moderate the impact of weight stigma on avoidance of exercise. Obesity (Silver Spring) 2011; 19 (4): 757-62.

33. Vartanian L, Shaprow J. Effects of weight stigma on exercise motivation and behavior: A preliminary investigation among college-aged females. J Health Psychol 2008; 13 (1): 131-8.

34. Major B, Hunger J, Bunyan D, Miller C. The ironic effects of weight stigma. J Exp Soc Psychol 2014; 51: 7480.

35. Schvey NA, Puhl RM, Brownell KD. The impact of weight stigma on caloric consumption. Obes (Silver Spring) 2011; 19 (10): 1957-62.

36. Major B, Eliezer D, Rieck H. The Psychological Weight of Weight Stigma. Soc Psychol Personal Sci 2012; 3 (6):
651-8.

37. Matthews KA, Salomon K, Kenyon K, Zhou F. Unfair Treatment, Discrimination, and Ambulatory Blood Pressure in Black and White Adolescents. Health Psychol 2005; 24 (3): 258-65.

38. Mold F, Forbes A. Patients' and professionals' experiences and perspectives of obesity in health-care settings: A synthesis of current research. Health Expect 2013; 16 (2): 119-42.

39. Puhl R, Peterson J, Luedicke J. Motivating or stigmatizing?; Public perceptions of weight-related language used by health providers. Int J Obes 2012; 37 (10): 6129.

40. Incollingo AC, Tomiyama AJ, Ward A. What does weight stigma smell like? Cross-modal influence of visual weight cues on olfaction. Int J Obes 2015; 39 (6): 10302.

41. Puhl R, Luedicke J, Lee Peterson J. Public reactions to obesity-related health campaigns: A randomized controlled trial. Am J Prev Med 2013; 45 (1): 36-48.

42. Teachman B, Brownell K. Implicit anti-fat bias among health professionals: Is anyone immune? Int J Obes 2001; 25: 1525-31.

43. Schwartz MB, Chambliss HO, Brownell KD, Blair SN, Billington C. Weight bias among health professionals specializing in obesity. Obes Res 2003; 11 (9): 1033-9.

44. Kaminsky J, Gadaleta D. A study of discrimination within the medical community as viewed by obese patients. Obes Surg 2002; 12 (1): 14-8.

45. Ward-Smith P, Peterson JA. Development of an instrument to assess nurse practitioner attitudes and beliefs about obesity. J Am Assoc Nurse Pract 2016; 28 (3): 125-9.

46. Brown I, Stride C, Psarou A, Brewins L, Thompson J. Management of obesity in primary care: Nurses' practices, beliefs and attitudes. J Adv Nurs 2007; 59 (4): 329-41.

47. Jay M, Kalet A, Ark T, McMacken M, Messito MJ, Richter R, et al. Physicians' attitudes about obesity and their associations with competency and specialty: a cross-sectional study. BMC Health Serv Res 2009; 9: 106.

48. Ferrante JM, Piasecki AK, Ohman-Strickland PA, Crabtree BF. Family physicians' practices and attitudes regarding care of extremely obese patients. Obesity (Silver Spring) 2009; 17 (9): 1710-6.

49. Wadden T, Anderson D, Foster GD, Bennett A, Steinberg C, Sarwer DB. Obese women's perceptions of their physicians' weight management attitudes and practices. Arch Fam Med 2000; 9 (9): 854-60. 